\title{
Outcomes Following Mid-Urethral Sling Placement in Patients with Intrinsic Sphincteric Deficiency: Comparison of Sparc and Monarc Slings
}

\author{
David E. Rapp, Fred E. Govier, Kathleen C. Kobashi
}

The Continence Center at Virginia Mason Medical Center, Seattle, Washington, USA

\begin{abstract}
Purpose: The treatment of patients with intrinsic sphincteric deficiency (ISD) remains difficult. It is theorized that differing vectors of support provided by retropubic versus transobturator mid-urethral sling routes may affect outcomes. We sought to compare outcomes of patients undergoing SPARC versus MONARC sling types in patients with Valsalva leak point pressures (VLPPs) below $60 \mathrm{~cm} \mathrm{H}_{2} \mathrm{O}$.

Materials and Methods: A retrospective review of female patients with stress urinary incontinence undergoing SPARC ${ }^{\mathrm{TM}}$ $(\mathrm{n}=97)$ or MONARC ${ }^{\mathrm{TM}}(\mathrm{n}=39)$ placement following urodynamic diagnosis of ISD was performed, with minimum 12month follow-up required. Outcomes were assessed using a questionnaire comprising validated incontinence questionnaires (UDI-6, IIQ-7) and additional items addressing satisfaction.

Results: Success rates of $76 \%$ and $77 \%$ were observed in the SPARC (mean follow-up 36 months) and MONARC (mean follow-up 32 months) cohorts, respectively ( $p>0.05)$. Superior UDI scores were demonstrated in the MONARC cohort $(3.8$ vs. $5.3, p=0.04)$ ), in contrast to similar IIQ scores across both groups $(3.7$ vs. $3.1, p>0.05)$. A deterioration in success rates was seen in both cohorts with more extended follow-up and with lower VLPPs. However, this finding was limited by low patient numbers in these cohorts. A complication rate of $7 \%$ and $3 \%$ was noted in SPARC and MONARC cohorts $(\mathrm{p}>0.05)$.

Conclusions: We observed no significant differences in subjective outcomes when comparing patients undergoing SPARC versus MONARC sling placement in the treatment of SUI with VLPP $<60 \mathrm{~cm} \mathrm{H}_{2} \mathrm{O}$. A deterioration in continence rates was seen with extended follow-up. These data may be affected by low patient numbers and related study power, in particular with more extended follow-up.
\end{abstract}

Key words: urinary incontinence; suburethral sling; treatment outcome; sphincteric deficiency

Int Braz J Urol. 2009; 35: 68-75

\section{INTRODUCTION}

The emergence of the integral theory and the mid-urethral sling (MUS) has resulted in an evolution of the understanding and treatment of stress urinary incontinence (SUI) (1). Multiple MUS types exist, with long-term outcomes supporting their use as a safe and efficacious treatment for SUI. Despite this success, a paucity of data exists to define the efficacy of these sling types in the specific treatment of intrinsic sphincteric deficiency (ISD).

As initially described, the MUS served to recreate a physiologic backboard beneath the urethra to prevent hypermobility and SUI. However, the non- 
obstructing support specific to MUS may not be ideal in patients with a fixed urethra and/or intrinsic sphincteric deficiency. In these cases, alternative sling types may be preferable. Indeed, it can be argued that the pubovaginal sling (PVS) is the gold standard in such cases, exhibiting important differences from MUS, such as immediate and secure sling fixation, a greater mesh width, and a bladder neck position. Supporting evidence has been reported in meta-analysis by Muller et al., demonstrating that in patients undergoing a TVT sling (Ethicon, Somerfield, NJ), urethral hypermobility is associated with increased efficacy in contrast to women with a fixed urethra who are at significant risk for procedure failure (2).

Despite such findings, a widespread acceptance of the MUS has been seen and such slings are commonly used in patients with not only type I SUI, but types II and III as well. With this in mind, research focusing on MUS outcomes in patients with ISD becomes increasingly important. Whereas some investigation has identified inferior cure rates following MUS in patients with Valsalva leak point pressures (VLPP) $<60 \mathrm{~cm} \mathrm{H}_{2} \mathrm{O}$, other investigators have found that pre-operative VLPP may not be predictive of outcome $(3,4)$.

Central to the issue of MUS outcomes in the treatment of ISD is the theoretical effect that sling vector may have on efficacy. Accordingly, it may be postulated that the more horizontal sling vector of the transobturator (TOT) sling approach may not provide equivalent obstruction and/or support when compared to retropubic (RP) sling types. Despite evidence suggesting that transobturator and retropubic MUS are associated with similar outcomes in a general population (5), it is unknown whether the differences in support vectors will be clinically significant in a focused population of patients with ISD. The purpose of this investigation was to assess for differences in patient-reported outcomes (PRO) following SPARC versus MONARC placement in a focused population of patients with VLPP $<60 \mathrm{~cm} \mathrm{H}_{2} \mathrm{O}$.

\section{MATERIALS AND METHODS}

A retrospective review of patients undergoing SPARC $^{\text {TM }}$ (American Medical Systems, Minnetonka,
$\mathrm{MN}$ ) or MONARC ${ }^{\mathrm{TM}}$ (American Medical Systems, Minnetonka, MN) mid-urethral sling placement following urodynamic diagnosis of ISD was performed. Review was performed using the prospectively collected Continence Center database at Virginia Mason Medical Center. All patients undergoing SPARC/ MONARC placement with a minimum follow-up of 12 months were included for data analysis; no other specific inclusion or exclusion criteria were used for patient identification.

As part of patient work-up for incontinence and database inclusion, all patients undergo standard history, general and focused urogynecologic physical examination, and videourodynamic (URD) evaluation. URD procedure and related terminology is performed in accordance with International Continence Society guidelines (6). VLPP measurement is performed in the standing position at cystometric bladder capacity. Gradual increases in abdominal straining are performed by patients with concurrent monitoring for urinary leakage via fluoroscopic visualization. VLPP is identified as the lowest intravesical pressure at the time or urinary leakage and is calculated as a differential value from baseline intravesical pressure.

Subjective satisfaction was assessed using a mailed questionnaire comprising separate validated incontinence and quality of life questionnaires (UDI6, IIQ-7), as well as additional items addressing global satisfaction and patient-perceived improvement. Outcome measures used for data analysis are detailed in Appendix-1. The primary study outcome assessed was dry rate by questionnaire response (Appendix-1, Question-1). Given the documented and significant effect that choice of outcome measure has on "success" rate, we also assessed a variety of related PRO. Accordingly, additional outcomes assessed included patients achieving $<1$ incontinence episode weekly, validated symptom score levels, overall patient satisfaction, and subjective percent improvement. Patient satisfaction and percent improvement are questionnaire items measured on a Likert scale. Overall outcomes were also presented using our previously described definition of success, which attempts to account for such known discrepancies in outcomes when measuring semi-objective variables such as incontinence episodes as compared to degrees of subjective patient-reported improvement. Therefore, 
we define success as $<1$ SUI episode per week or $>70 \%$ subjective improvement in those patients with $>1$ SUI episode per week. Additional focus was placed on assessing for differences in the rate and type of surgical complications.

Our technique for SPARC placement is previously described (7). MONARC sling placement is performed using standard technique, as originally described by Delorme (8). Surgical procedures were performed by one of two surgeons (FG, KK). Procedures were performed under general or spinal anesthesia per patient preference. Given the referral pattern characteristic of our institution, patients are admitted overnight to allow for observation and avoid lengthy travel in the immediate post-operative hours. As part of this protocol, both patients undergoing sling with and without concurrent pelvic organ prolapse (POP) repair receive IV antibiotics and vaginal packing. The Foley catheter and vaginal packing are removed on the following morning prior to discharge. The study was approved by the Virginia Mason Medical Center Institutional Review Board. Z-test for proportions was used to compare outcome rates. Student's " $t$ " test was used for comparative data. Each analysis was structured as a two-tailed test at the $\alpha=0.05$ level.

\section{RESULTS}

Retrospective review identified 107 (SPARC) and 43 (MONARC) patients achieving 12-month minimum follow-up. Of this population, 97 (91\%) and $39(91 \%)$ of patients completed follow-up questionnaires and were included for per-protocol data analysis.

A complete list of patient demographics and characteristics is provided in Table-1. Mean followup was significantly longer in the SPARC cohort ( 36 versus 32 months) $(\mathrm{p}=0.03)$. In addition, mean VLPP was lower in the SPARC cohort (43 versus 49 $\left.\mathrm{cm} \mathrm{H}_{2} \mathrm{O}\right)(\mathrm{p}<0.01)$. No other significant differences were identified in comparison of baseline cohort characteristics.

Subjective outcomes following MUS placement are detailed in Tables 2-3. Dry rates of 29\% and $41 \%$ were observed in the SPARC and MONARC cohorts, respectively. An additional $39 \%$ and $28 \%$ of patients reported incontinence episodes $<1$ /weekly, respectively. Based on the previously described definition of success, $76 \%$ and $77 \%$ of procedures were considered successful in these cohorts, respectively. Comparison of each of these outcomes revealed no

Table 1 - Patient demographics and characteristics.

\begin{tabular}{lccc}
\hline & Sparc $(\mathbf{N}=\mathbf{9 7})$ & Monarc $(\mathbf{N}=\mathbf{3 9})$ & p Value \\
\hline Age $(\mathrm{y} \pm \mathrm{SD})$ & $64 \pm 11$ & $61 \pm 12$ & $\mathrm{NS}$ \\
Follow-up (y $\pm \mathrm{SD})$ & $2.9 \pm 0.9$ & $1.8 \pm 0.8$ & 0.03 \\
VLPP (cm H20 $\pm \mathrm{SD})$ & $43 \pm 11$ & $49 \pm 14$ & $<0.01$ \\
Parity $(\mathrm{n} \pm \mathrm{SD})$ & $2.5 \pm 1.4$ & $2.5 \pm 1.2$ & $\mathrm{NS}$ \\
Previous surgeries (N) & & & \\
$\quad$ Hysterectomy & $42(43 \%)$ & $15(38 \%)$ & $\mathrm{NS}$ \\
$\quad 17(18 \%)$ & $6(15 \%)$ & $\mathrm{NS}$ \\
$\quad$ Anti-incontinence & $7(7 \%)$ & $5(13 \%)$ & $\mathrm{NS}$ \\
Prolapse repair & $26(27 \%)$ & $14(36 \%)$ & $\mathrm{NS}$ \\
$\quad$ Ancurrent surgeries (N) & $21(22 \%)$ & $6(15 \%)$ & $\mathrm{NS}$ \\
$\quad$ Posterior colporrhaphy & $2(2 \%)$ & $2(5 \%)$ & $\mathrm{NS}$ \\
$\quad$ Vault suspension & $5(7 \%)$ & $1(3 \%)$ & NS \\
Complications (N) & & & \\
\hline
\end{tabular}

$N S=$ not significant $(p>0.05) . V L P P=$ Valsalva leak point pressure, Student's " $t$ " test was used for analysis of comparative data (age, follow-up, VLPP, parity); Z-test for proportions was used for remaining comparisons (surgeries, complications). 
Table 2 - Outcomes following mid-urethral sling placement.

\begin{tabular}{lccc}
\hline Outcome & Sparc $(\mathbf{n}=\mathbf{9 7})$ & Monarc $(\mathbf{n}=\mathbf{3 9})$ & p Value \\
\hline Incontinence frequency & & & \\
$\quad$ Dry (no leakage) & $28(29 \%)$ & $16(41 \%)$ & NS \\
$\quad<1$ episode/week & $38(39 \%)$ & $11(28 \%)$ & NS \\
\% Improvement $(\geq 70 \%)$ & $66(68 \%)$ & $26(67 \%)$ & NS \\
Success & $74(76 \%)$ & $30(77 \%)$ & NS \\
Satisfaction ( $\geq 7)$ & $63(65 \%)$ & $26(67 \%)$ & NS \\
Recommend (Yes) & $65(67 \%)$ & $28(72 \%)$ & NS \\
Repeat (Yes) & $69(71 \%)$ & $30(77 \%)$ & NS \\
UDI-6* & $5.3(3.9)$ & $3.8(3.5)$ & 0.04 \\
IIQ-7* & $3.7(3.6)$ & $3.1(4.6)$ & NS \\
\hline
\end{tabular}

* Figures are listed as mean score (standard deviation). NS = not significant $(p>0.05)$. Student's t test was used for analysis of comparative data (UDI/IIQ); Z-test for proportions used for remaining comparisons. UDI-6 = validated incontinence questionnaire; $I I Q-7$ = quality of life questionnaire.

statistically significant differences. Intention to treat analysis of continence and success rates was carried out assuming all questionnaire non-responders to be failures. In this analysis, dry rates of $26 \%$ versus $37 \%$ and success rates of $69 \%$ versus $70 \%$ were seen in the SPARC and MONARC cohorts, respectively. Again, these comparisons revealed no statistically significant differences.

As described, significant differences in mean VLPP and mean follow-up were identified in the comparison of SPARC and MONARC cohorts. Accordingly, cohorts were further stratified by VLPP and follow-up length in an attempt to assess for outcome differences influenced by these baseline differences (Table-3). No significant differences in continence rates were identified in comparing MUS types across these stratification points. However, a deterioration in success rates was observed in both cohorts with more extended follow-up and with lower VLPPs. This finding was limited by low patient numbers in these cohorts.

Table 3 - Incontinence rates following mid-urethral sling placement.

\begin{tabular}{|c|c|c|c|c|c|c|c|c|c|}
\hline \multicolumn{5}{|c|}{ Sparc $(\mathbf{N}=97)$} & \multicolumn{5}{|c|}{$\operatorname{Monarc}(\mathbf{N}=39)$} \\
\hline $\operatorname{VLPP}\left(\mathrm{cm} \mathrm{H}_{2} \mathrm{O}\right)$ & N of Pts & Never & $<1 /$ week & Success & N of Pts & Never & $<1 /$ week & Success & p Value \\
\hline $50-59$ & 36 & 12 & 14 & $31(86)$ & 26 & 14 & 7 & $22(85)$ & NS \\
\hline $40-49$ & 29 & 10 & 11 & $21(72)$ & 6 & 2 & 1 & $4(66)$ & NS \\
\hline $30-39$ & 19 & 4 & 7 & $12(63)$ & 5 & 0 & 2 & $3(60)$ & NS \\
\hline$<30$ & 13 & 2 & 6 & $12(50)$ & 2 & 0 & 1 & $1(50)$ & NS \\
\hline \multicolumn{10}{|l|}{ Follow-up (years) } \\
\hline $1-2$ & 17 & 3 & 9 & $13(76)$ & 25 & 13 & 5 & $20(80)$ & NS \\
\hline $2-3$ & 34 & 10 & 14 & $25(74)$ & 10 & 2 & 5 & $8(80)$ & NS \\
\hline $3-4$ & 38 & 13 & 14 & $33(87)$ & 3 & 1 & 1 & $2(67)$ & NS \\
\hline $4+$ & 8 & 2 & 1 & $3(40)$ & 1 & 0 & 0 & $0(40)$ & $\mathrm{NS}$ \\
\hline
\end{tabular}

Data represents number of patients (percentage of total). $N S=$ non-significant $(p>0.05) ;$ Pts $=$ patients; Z-test for proportions used for comparisons. $V L P P=$ Valsalva leak point pressure . 
Comparison of UDI-6 and IIQ-7 questionnaire scores was also performed. UDI scores were superior in the MONARC cohort $(\mathrm{p}=0.04)$, whereas IIQ scores did not demonstrate significant differences when comparing MUS types. Finally, an overall complication rate of $7 \%(7 / 97)$ and 3\% $(1 / 39)$ was observed in the SPARC and MONARC cohorts, respectively. Vaginal mesh extrusion $(n=2$, SPARC; $\mathrm{n}=0$ MONARC) and blood loss requiring transfusion $(n=2$, SPARC; $n=1$ MONARC) were the most common complications. Vaginal extrusions were each treated with operative reclosure under sedation. Additional complications in the SPARC cohort included persistent granulation tissue/suture granuloma requiring local excision. This complication was considered to be associated with concomitant POP repair, although it is reported to completely present our data. No trends in complication incidence were noted in this comparison.

\section{COMMENTS}

The primary finding of this study is the demonstration of similar subjective outcomes in SPARC and MONARC cohorts with baseline VLPP $<60 \mathrm{~cm} \mathrm{H}_{2} \mathrm{O}$. In the comparison of multiple PRO measures, findings were similar between the two cohorts. Interestingly, a superior patient-reported dry rate was associated with MONARC placement, although this finding failed to achieve statistical significance. Given the limited study population, we cannot associate any clinical difference with this finding. In addition, superiority of UDI scores was seen in the MONARC cohort, although this finding was not reproduced in comparison of IIQ scores.

Given the variety of MUS types currently in use, more recent research focus has been placed on assessing for potential clinical differences in comparative outcomes. In comparison of RP and TOT sling types in a generalized SUI population, significant randomized investigation would suggest comparable subjective and objective outcomes with short-term follow-up $(5,9,10)$. Despite these findings, the treatment of ISD may represent a more complex undertaking. Indeed, non-comparative investigation has demonstrated that patients with maximum urethral closing pressure (MUCP) less than $40 \mathrm{~cm} \mathrm{H}_{2} \mathrm{O}$ are associated with a higher failure rate following TOT as compared to that found with higher closing pressures (11). In a similar fashion, lower VLPPs are associated with inferior outcomes following TOT (3). However, other investigation focusing only on RP sling types suggests that VLPP may not predict for outcome following MUS surgery $(4,12,13)$. Therefore, it becomes crucial that the urologic community assess whether differing sling approaches (e.g. TOT versus RP) are equally efficacious in ISD cohorts.

Theoretically, it is possible that the differing sling vectors of RP and TOT types will be associated with clinical differences that are only evident in patients with more severe incontinence as characteristic of ISD. Anatomic study supports the concept of differing vectors, with a more vertical, U-shaped vector characteristic of the RP slings, in contrast to the more horizontal, hammock-shaped vector of the TOT types (14). Additional anatomical differences are suggested by sonographic study demonstrating a more proximal position of the TOT slings at both rest and Valsalva, in comparison to RP types (15). Accordingly, a more circumferential compression of the urethra may be afforded through RP slings. In contrast, the lateral vector of TOT slings may not allow for equivalent suburethral tensioning (16). Clinical evidence supports this theory, in which progressive intra-operative tensioning of TOT slings was unable to stop cough test induced urinary leakage (17). In contrast, a negative cough test was achieved following immediate removal of the TOT slings and subsequent replacement by a RP sling in a "tensionless" fashion.

Limited clinical investigation has specifically focused on comparison of success rates in RP versus TOT cohorts with documented ISD. Miller et al. demonstrated that patients with a MUCP $\leq 42 \mathrm{~cm}$ $\mathrm{H}_{2} \mathrm{O}$ had a relative risk for procedure failure of 5.89 following MONARC, as compared to a similar cohort of patients undergoing TVT (18).

A paucity of additional directed investigation exists. However, further indirect evidence supporting the clinical importance of vector differences between $\mathrm{RP}$ and TOT slings is gleaned through analysis of persistent and de novo urge urinary incontinence (UUI) 
and voiding dysfunction following MUS placement. Accordingly, Botros et al. demonstrated a reduced rate of de novo UUI, as well as an increased chance of resolution of pre-operative UUI with TOT versus RP sling types (16). Concurrently, Dietz and colleagues identified a lower incidence of voiding dysfunction symptomatology with MONARC (versus TVT), concluding that the MONARC sling may be less obstructive when compared to RP types (15). Although such data cannot be extrapolated to continence outcomes, they serve to further suggest that vector (and associated tensioning or positional) differences may exist between RP and TOT slings and that these differences may have clinical implications.

The authors acknowledge certain weaknesses in our study. First, the retrospective nature is a limitation to our study design. Second, the difference in observed follow-up length may bias outcomes, as MONARC patients may be associated with an increased failure rate given longer follow-up. It should be emphasized, however, that the previously described deterioration in outcomes following transobturator sling reported by other groups occurred with far shorter follow-up than that presented in our investigation $(18,19)$. Further, several PRO measures reported are not empirically validated. Nonetheless, our data are strengthened by the use of a comprehensive collection of PRO measures commonly used in incontinence research.

Notably, the higher baseline VLPP observed in the MONARC cohort may bias results in favor of these patients. While such differences may be within error characteristic of UDY VLPP measurement and may not be clinically meaningful, we believe this difference is important to detail. Certainly, this difference reflects a selection bias, as surgeons were not blinded to pre-operative VLPP and favored a retropubic approach in patients with more severe ISD. Subset analysis attempting to control for VLPP and follow-up differences found no differences. Most importantly, however, these and other conclusions are limited by a small study population that is particularly notable with extended follow-up. Despite these limitations, we believe that our data most importantly serve to suggest that prospective randomized study is needed, given the limited and conflicting data that exists, and the significant popularity of the MUS in the current treatment algorithm for SUI.
A secondary finding to our study is a deterioration in continence rates observed irrespective of sling type in ISD cohorts with extended followup. Certainly, the widespread use of MUS is accompanied by mid- and long-term data suggesting good continence outcomes. In such reports, cure rates range from $80 \%-95 \%$ across all MUS types and include follow-up extending to 83 months (20). We have previously suggested that strict long-term continence rates may not consistently approach these rates, with a strict dry rate of $34 \%$ being identified in our experience following SPARC with long-term follow-up (minimum 24 months, mean 36 months) (7). Both the present data and additional series would again suggest that dry and success rates may experience significant deterioration with long-term follow-up and may be particularly problematic in patients with ISD (19). Foremost, this finding would underscore the need to critically assess long-term dry rates in patients following MUS placement and to further focus such investigation on outcomes in a defined population with ISD. Indeed, it may be possible that both RP and TOT sling types are not optimal for patients with low VLPP and that consideration of other sling types (e.g. PVS) in this complex cohort should be revisited.

\section{CONCLUSION}

We identified no significant differences in subjective outcomes when comparing patients undergoing SPARC versus MONARC sling placement in the treatment of SUI with VLPP $<60 \mathrm{~cm} \mathrm{H}_{2} \mathrm{O}$. A deterioration in continence rates was seen with extended follow-up. These data may be affected by low patient numbers and related study power, in particular with more extended follow-up. Foremost, this investigation would highlight the importance for future prospective and randomized study assessing retropubic and transobturator MUS outcomes in patients with ISD is important.

\section{CONFLICT OF INTEREST}

None declared. 
Appendix - Selected outcomes measures from incontinence questionnaire.

1. Do you leak when you cough, sneeze, or perform physical activities? [Incontinence Frequency] A) never $B$ ) $<1 /$ week $C$ ) once/day D) always $E$ ) not sure

2. How much improved is your urinary leakage now compared to before the surgery? [\% Improvement] $100 \%$ better, $90 \%, 80 \%, 70 \%, 60 \%, 50 \%, 40 \%, 30 \%, 20 \%, 10 \%$ better, the same, worse

3. Overall, how satisfied are you with the results of your sling surgery? [Satisfaction]

0 (not satisfied), 1, 2, 3, 4, 5, 6, 7, 8, 9, 10 (very satisfied)

4. Knowing what you know now, would you have the sling surgery again? [Repeat] A) yes B) no C) not sure

5. Would you recommend the sling surgery to a friend? [Recommend] A) yes B) no, C) not sure

[ ] Indicate outcome measure assessed by question

\section{REFERENCES}

1. Petros PE, Ulmsten UI: An integral theory and its method for the diagnosis and management of female urinary incontinence. Scand J Urol Nephrol Suppl. 1993; 153: 1-93.

2. Muller M, Koebele A, Deval B: Determinants of success and recurrence after suburetral free tape procedure for female urinary incontinence. J Gynecol Obstet Biol Reprod (Paris). 2007; 36: 19-29.

3. O'Connor RC, Nanigian DK, Lyon MB, Ellison LM, Bales GT, Stone AR: Early outcomes of mid-urethral slings for female stress urinary incontinence stratified by valsalva leak point pressure. Neurourol Urodyn. 2006; 25: 685-8.

4. Cetinel B, Demirkesen O, Onal B, Akkus E, Alan C, Can G: Are there any factors predicting the cure and complication rates of tension-free vaginal tape? Int Urogynecol J Pelvic Floor Dysfunct. 2004; 15: 18893.

5. Tseng LH, Wang AC, Lin YH, Li SJ, Ko YJ: Randomized comparison of the suprapubic arc sling procedure vs tension-free vaginal taping for stress incontinent women. Int Urogynecol J Pelvic Floor Dysfunct. 2005; 16: 230-5.

6. Abrams P, Cardozo L, Fall M, Griffiths D, Rosier P, Ulmsten $U$, et al.: The standardisation of terminology of lower urinary tract function: report from the Standardisation Sub-committee of the International
Continence Society. Neurourol Urodyn. 2002; 21: 167-78.

7. Nazemi TM, Yamada B, Govier FE, Kuznetsov DD, Kodama K, Kobashi KC: Minimum 24-month followup of the sling for the treatment of stress urinary incontinence. J Urol. 2008; 179: 596-9.

8. Delorme E, Droupy S, de Tayrac R, Delmas V: Transobturator tape (Uratape): a new minimally-invasive procedure to treat female urinary incontinence. Eur Urol. 2004; 45: 203-7.

9. Liapis A, Bakas P, Creatsas G: Monarc vs TVT-O for the treatment of primary stress incontinence: a randomized study. Int Urogynecol J Pelvic Floor Dysfunct. 2008; 19: 185-90.

10. Barry C, Lim YN, Muller R, Hitchins S, Corstiaans A, Foote A, et al.: A multi-centre, randomised clinical control trial comparing the retropubic (RP) approach versus the transobturator approach (TO) for tensionfree, suburethral sling treatment of urodynamic stress incontinence: the TORP study. Int Urogynecol J Pelvic Floor Dysfunct. 2008; 19: 171-8.

11. Guerette NL, Bena JF, Davila G: Transobturator slings for stress incontinence: using urodynamic parameters to predict outcomes. Int Urogynecol J Pelvic Floor Dysfunct. 2008; 19: 97-102.

12. Rodríguez LV, de Almeida F, Dorey F, Raz S: Does Valsalva leak point pressure predict outcome after the distal urethral polypropylene sling? Role of urodynamics in the sling era. J Urol. 2004; 172: 210-4. 
13. Abdel-Hady el-S, Constantine G: Outcome of the use of tension-free vaginal tape in women with mixed urinary incontinence, previous failed surgery, or low valsalva pressure. J Obstet Gynaecol Res. 2005; 31: 38-42.

14. Whiteside JL, Walters MD: Anatomy of the obturator region: relations to a trans-obturator sling. Int Urogynecol J Pelvic Floor Dysfunct. 2004; 15: 223-6.

15. Dietz HP, Barry C, Lim Y, Rane A: TVT vs Monarc: a comparative study. Int Urogynecol J Pelvic Floor Dysfunct. 2006; 17: 566-9.

16. Botros SM, Miller JJ, Goldberg RP, Gandhi S, Akl M, Beaumont JL, et al.: Detrusor overactivity and urge urinary incontinence following trans obturator versus midurethral slings. Neurourol Urodyn. 2007; 26: $42-$ 5.
17. Mukai M, Aboujaoude R, Culligan PJ: Two cases illustrating a potential difference between transobturator and retropubic slings. Int Urogynecol J Pelvic Floor Dysfunct. 2007; 18: 967-9.

18. Miller JJ, Botros SM, Akl MN, Aschkenazi SO, Beaumont JL, Goldberg RP, et al.: Is transobturator tape as effective as tension-free vaginal tape in patients with borderline maximum urethral closure pressure? Am J Obstet Gynecol. 2006; 195: 1799-804.

19. Jeon MJ, Jung HJ, Chung SM, Kim SK, Bai SW: Comparison of the treatment outcome of pubovaginal sling, tension-free vaginal tape, and transobturator tape for stress urinary incontinence with intrinsic sphincter deficiency. Am J Obstet Gynecol. 2008; 199: 76-7.

20. Rapp DE, Kobashi KC: The evolution of midurethral slings. Nat Clin Pract Urol. 2008; 5: 194-201.

Accepted after revision:

October 2, 2008

\section{Correspondence address:}

Dr. David E. Rapp

Virginia Mason Medical Center

1100 9th AVE C-7 URO

Seattle, WA, 98111, USA

Fax: + 1206 223-7650

E-mail: derapp@yahoo.com

\section{EDITORIAL COMMENT}

This is an interesting paper comparing suprapubic versus transobturatory slings for the treatment of females with stress urinary incontinence (SUI) due to intrinsic sphincter deficiency (ISD). Even though this is a retrospective study it allows some interesting conclusions. Few studies in the literature compared the Monarc and Sparc slings for the treatment of females with SUI due to ISD. In particular, few studies compared the present type of material. The majority compares TVT and TVTO. Therefore, this study is important because shows that the transobturatory approach has the same efficacy of the suprapubic approach even in women with ISD. This also has been demonstrated with the TVTO. On the other hand, the complication rates are also very similar showing the two approaches seems to be equivalent.

It would be very useful to have a randomized controlled trial comparing these two approaches to treat female SUI.

\author{
Dr. Flavio Trigo Rocha \\ Division of Urology \\ University of Sao Paulo, USP \\ Sao Paulo, SP, Brazil \\ E-mail: flaviotrigo@uol.com.br
}

